Harant, Jochen:

Some news about the independence number of a graph

URN: $\quad$ urn:nbn:de:gbv:ilm1-2020200043

\begin{tabular}{|c|c|}
\hline Original published in: & $\begin{array}{l}\text { Discussiones mathematicae. Graph theory / Uniwersytet Zielonogórski } \\
\text { Wydział Matematyki, Informatyki i Ekonometrii. - Warsaw : De Gruyter } \\
\text { Open. - } 20 \text { (2000), 1, p. 71-79. }\end{array}$ \\
\hline Original published: & 2000 \\
\hline ISSN: & $2083-5892$ \\
\hline DOI: & 10.7151/dmgt.1107 \\
\hline \multirow[t]{2}{*}{ [Visited: } & 2020-01-17] \\
\hline & $\begin{array}{l}\text { This work is licensed under a Creative Commons Attribution- } \\
\text { NonCommercial-NoDerivatives } 3.0 \text { Unported license. } \\
\text { To view a copy of this license, visit } \\
\text { http://creativecommons.org/licenses/BY-NC-ND/3.0/ }\end{array}$ \\
\hline
\end{tabular}


Discussiones Mathematicae

Graph Theory 20 (2000) 71-79

\title{
SOME NEWS ABOUT THE INDEPENDENCE NUMBER OF A GRAPH
}

\author{
JOCHEN HARANT \\ Department of Mathematics, Technical University of Ilmenau \\ D-98684 Ilmenau, Germany
}

\begin{abstract}
For a finite undirected graph $G$ on $n$ vertices some continuous optimization problems taken over the $n$-dimensional cube are presented and it is proved that their optimum values equal the independence number of $G$.
\end{abstract}

Keywords: graph, independence.

1991 Mathematical Subject Classification: 05C35.

\section{Introduction and Results}

Let $G$ be a finite simple and undirected graph on $V(G)=\{1,2, \ldots, n\}$ with its edge set $E(G)$. A subset $I$ of $V(G)$, such that the subgraph of $G$ induced by $I$ is edgeless, is called an independent set of $G$, and the maximum cardinality of an independent set of $G$ is named the independence number $\alpha(G)$ of $G$. $N(i)$ and $d_{i}$ denote the set and the number of neighbours of $i \in V(G)$ in $G$, respectively, and let $\Delta(G)=\max \left\{d_{i} \mid i \in V(G)\right\}$ and $C^{n}=\left\{\left(x_{1}, x_{2}, \ldots, x_{n}\right) \mid 0 \leq x_{i} \leq 1, i=1,2, \ldots, n\right\}$. For events $A$ and $B$ and for a random variable $Z$ of an arbitrary random space, $P(A)$, $P(A \mid B)$, and $\mathcal{E}(Z)$ denote the probability of $A$, the conditional probability of $A$ given $B$, and the expectation of $Z$, respectively. Since the computation of $\alpha(G)$ is difficult (INDEPENDENT SET is an NP-complete problem; see [6]), much work was done to establish bounds on $\alpha(G)$ (e.g., see $[1,3,4,5,8,10,12,13,14,15,16,17])$, to find efficient algorithms forming a large independent set of $G$ (e.g., see $[2,7,8,9,10,12]$ ), or to replace the combinatorial optimization problem to determine $\alpha(G)$ by a continuous one 
(e.g., see $[9,11])$. The last approach leads to bounds on $\alpha(G)$ as well as to efficient algorithms (e.g., see $[8,9]$ ). In the present paper some new continuous optimization problems taken over $C^{n}$ are presented and it is proved that their optimum values equal $\alpha(G)$. Theorem 1 gives a remarkable result of T.S. Motzkin and E.G. Straus [11] and Theorem 2 is proved in [9].

\section{Theorem 1.}

$\alpha(G)=\max _{(0,0, \ldots, 0) \neq\left(x_{1}, x_{2}, \ldots, x_{n}\right) \in C^{n}} \frac{\left(\sum_{i \in V(G)} x_{i}\right)^{2}}{\sum_{i \in V(G)} x_{i}^{2}+2 \sum_{i j \in E(G)} x_{i} x_{j}}$.

Theorem 2. $\alpha(G)=\max _{\left(x_{1}, x_{2}, \ldots, x_{n}\right) \in C^{n}} \sum_{i \in V(G)}\left(x_{i} \prod_{j \in N(i)}\left(1-x_{j}\right)\right)$.

A classical lower bound on $\alpha(G)$ due to Y. Caro and V.K. Wei [3, 17] is given by the following theorem.

Theorem 3. $\alpha(G) \geq \sum_{i \in V(G)} \frac{1}{1+d_{i}}$.

The next Theorems 4, 5,6, and 7 are the main results of the present paper.

Theorem 4. $\alpha(G)=\underset{\left(x_{1}, x_{2}, \ldots, x_{n}\right) \in C^{n}}{\max } e_{G}\left(x_{1}, x_{2}, \ldots, x_{n}\right)$, where $e_{G}\left(x_{1}, x_{2}, \ldots, x_{n}\right)=\sum_{i \in V(G)}\left(\frac{x_{i}}{1+\sum_{j \in N(i)} x_{j}}+\frac{\left(1-x_{i}\right) \prod_{j \in N(i)}\left(1-x_{j}\right)}{1+\sum_{j \in N(i)} \prod_{l \in N(j) \backslash(N(i) \cup\{i\})}\left(1-x_{l}\right)}\right)$.

Theorem 5. $\alpha(G)=\max _{\left(x_{1}, x_{2}, \ldots, x_{n}\right) \in C^{n}} f_{G}\left(x_{1}, x_{2}, \ldots, x_{n}\right)$, where $f_{G}\left(x_{1}, x_{2}, \ldots, x_{n}\right)=\sum_{i \in V(G)}\left(x_{i}+\frac{\left(1-x_{i}\right)}{1+\sum_{j \in N(i)} \prod_{l \in N(j) \backslash(N(i) \cup\{i\})}\left(1-x_{j}\right)}\right)-\sum_{i j \in E(G)} x_{i} x_{j}$.

The following Theorem 6 looks more "complicate", but it is "stronger" than Theorem 4 and Theorem 5 (see Remark 1).

Theorem 6. $\alpha(G)=\max _{\left(x_{1}, x_{2}, \ldots, x_{n}\right) \in C^{n}} g_{G}\left(x_{1}, x_{2}, \ldots, x_{n}\right)$, where $g_{G}\left(x_{1}, x_{2}, \ldots, x_{n}\right)=\sum_{i \in V(G)}\left(\left(x_{i}+\frac{1-x_{i}}{1+\sum_{j \in N(i)} \prod_{l \in N(j) \backslash(N(i) \cup\{i\})}\left(1-x_{l}\right)}\right) \prod_{j \in N(i)}\left(1-x_{j}\right)\right)$ 
$+\sum_{i \in V^{\prime}} \frac{x_{i}\left(1-\prod_{j \in N(i)}\left(1-x_{j}\right)\right)^{2}}{1-\prod_{j \in N(i)}\left(1-x_{j}\right)+\sum_{j \in N(i)} x_{j}}$ and $V^{\prime}=\left\{i \in V(G) \mid \sum_{j \in N(i)} x_{j}>0\right\}$.

A "weaker" (see Remark 1), but a more "transparent" and (see Remark 2) an "algorithmically realizable" version of Theorem 5 is the following one.

Theorem 7. $\alpha(G)=\max _{\left(x_{1}, x_{2}, \ldots, x_{n}\right) \in C^{n}} h_{G}\left(x_{1}, x_{2}, \ldots, x_{n}\right)$, where $h_{G}\left(x_{1}, x_{2}, \ldots, x_{n}\right)=\sum_{i \in V(G)} x_{i}-\sum_{i j \in E(G)} x_{i} x_{j}$.

\section{Proofs}

Throughout the proofs we will use the well-known fact that for a random subset $M$ of a given finite set $N$,

$$
\mathcal{E}(|M|)=\sum_{y \in N} P(y \in M)=\sum_{k=0}^{|N|} k P(|M|=k) .
$$

Let $I$ be a maximum independent set of $G$ and let $x_{i}^{*}=1$ if $i \in I$ and $x_{i}^{*}=0$ if $i \notin I$. Since $\left(1-x_{i}^{*}\right) \prod_{j \in N(i)}\left(1-x_{j}^{*}\right)=0$ for $i \in V(G)$ and $\sum_{i j \in E(G)} x_{i}^{*} x_{j}^{*}=0$, we obtain

Lemma 1. $\alpha(G)=e_{G}\left(x_{1}^{*}, x_{2}^{*}, \ldots, x_{n}^{*}\right)=f_{G}\left(x_{1}^{*}, x_{2}^{*}, \ldots, x_{n}^{*}\right)=g_{G}\left(x_{1}^{*}, x_{2}^{*}, \ldots, x_{n}^{*}\right)$ $=h_{G}\left(x_{1}^{*}, x_{2}^{*}, \ldots, x_{n}^{*}\right)$.

With Lemma 1, it is clear that Theorem 7 follows from Theorem 5 .

Now, let $\left(x_{1}, x_{2}, \ldots, x_{n}\right)$ be an arbitrary member of $C^{n}$. We form a set $X \subseteq$ $V(G)$ by random and independent choice of $i \in V(G)$, where $P(i \in X)=x_{i}$. Let $H_{1}, H_{2}$, and $H_{3}$ be the subgraph of $G$ induced by the vertices of $X$, by the vertices $i \in X$ with $N(i) \cap X \neq \emptyset$, and by the vertices $i \notin X$ with $N(i) \cap X=\emptyset$, respectively. Furthermore, let $Y$ be a smallest subset of $V\left(H_{2}\right)$ covering all edges of $H_{2}$, i.e., the graph induced by $V\left(H_{2}\right)-Y$ is edgeless, and let $I_{1}$ and $I_{3}$ be a maximum independent set of $H_{1}$ and $H_{3}$, respectively. It can be seen easily that $|Y|=\left|V\left(H_{2}\right)\right|-\alpha\left(H_{2}\right),|Y| \leq\left|E\left(H_{2}\right)\right|$ and that $(X-Y) \cup I_{3}$ and $I_{1} \cup I_{3}$ are independent sets of $G$. Because of these remarks and the property of the expectation to be an average value, we have Lemma 2 as follows. 
Lemma 2. $\alpha(G) \geq \mathcal{E}(|X-Y|)+\mathcal{E}\left(\alpha\left(H_{3}\right)\right), \alpha(G) \geq \mathcal{E}\left(\alpha\left(H_{1}\right)\right)+\mathcal{E}\left(\alpha\left(H_{3}\right)\right)$, $\mathcal{E}(|X-Y|)=\mathcal{E}(|X|)-\mathcal{E}(|Y|) \geq \mathcal{E}(|X|)-\mathcal{E}\left(\left|E\left(H_{2}\right)\right|\right)$, and $\mathcal{E}(|X-Y|)=\mathcal{E}(|X|)-\mathcal{E}\left(\left|V\left(H_{2}\right)\right|\right)+\mathcal{E}\left(\alpha\left(H_{2}\right)\right)$.

Lower bounds on $\mathcal{E}\left(\alpha\left(H_{1}\right)\right), \mathcal{E}\left(\alpha\left(H_{2}\right)\right)$, and $\mathcal{E}\left(\alpha\left(H_{3}\right)\right)$ are given in Lemma 3.

Lemma 3. $\mathcal{E}\left(\alpha\left(H_{1}\right)\right) \geq \sum_{i \in V(G)} \frac{x_{i}}{1+\sum_{j \in N(i)} x_{j}}$,

$\mathcal{E}\left(\alpha\left(H_{2}\right)\right) \geq \sum_{i \in V^{\prime}} \frac{x_{i}\left(1-\prod_{j \in N(i)}\left(1-x_{j}\right)\right)^{2}}{1-\prod_{j \in N(i)}\left(1-x_{j}\right)+\sum_{j \in N(i)} x_{j}}$, where $V^{\prime}=\left\{i \in V(G) \mid \sum_{j \in N(i)} x_{j}>0\right\}$,

and

$\mathcal{E}\left(\alpha\left(H_{3}\right)\right) \geq \sum_{i \in V(G)} \frac{\left(1-x_{i}\right) \prod_{j \in N(i)}\left(1-x_{j}\right)}{1+\sum_{j \in N(i)} \prod_{l \in N(j) \backslash(N(i) \cup\{i\})}\left(1-x_{l}\right)}$.

Proof. For $i \in V(G)$ define the random variable $Z_{i}^{1}$ with $Z_{i}^{1}=\frac{1}{1+k}$ if $i \in X$ and $|N(i) \cap X|=k \geq 0$, and $Z_{i}^{1}=0$ if $i \notin X$. Using Theorem 3,

$$
\begin{aligned}
& \mathcal{E}\left(\alpha\left(H_{1}\right)\right) \geq \mathcal{E}\left(\sum_{i \in V(G)} Z_{i}^{1}\right)=\sum_{i \in V(G)} \mathcal{E}\left(Z_{i}^{1}\right) \\
& =\sum_{i \in V(G)} \sum_{k=0}^{d_{i}} \frac{1}{1+k} P(i \in X \text { and }|N(i) \cap X|=k) \\
& =\sum_{i \in V(G)} \sum_{k=0}^{d_{i}} \frac{1}{1+k} P(i \in X) P(|N(i) \cap X|=k) \\
& \left.=\sum_{i \in V(G)} x_{i} \sum_{k=0}^{d_{i}} \frac{1}{1+k} P(|N(i) \cap X|=k)\right) .
\end{aligned}
$$

For $i \in V(G)$ we have $\sum_{k=0}^{d_{i}} P(|N(i) \cap X|=k)=1$. With Jensen's inequality $\sum_{l=1}^{m} \tau_{l} \phi\left(y_{l}\right) \geq \phi\left(\sum_{l=1}^{m} \tau_{l} y_{l}\right)$ for any convex function $\phi$ and any $\tau_{l} \geq 0$ for $l=$ $1,2, \ldots, m$ with $\sum_{l=1}^{m} \tau_{l}=1$,

$\mathcal{E}\left(\alpha\left(H_{1}\right)\right) \geq \sum_{i \in V(G)} x_{i} \frac{1}{1+\sum_{k=0}^{d_{i}} k P(|N(i) \cap X|=k)}=\sum_{i \in V(G)} \frac{x_{i}}{1+\sum_{j \in N(i)} x_{j}}$.

Now, let $V^{\prime}=\left\{i \in V(G) \mid \sum_{j \in N(i)} x_{j}>0\right\}$. For $i \in V(G)$ let $Z_{i}^{2}$ be the random variable with $Z_{i}^{2}=\frac{1}{1+k}$ if $i \in X$ and $|N(i) \cap X|=k \geq 1$, and $Z_{i}^{2}=0$ otherwise. Then, 


$$
\begin{aligned}
& \mathcal{E}\left(\alpha\left(H_{2}\right)\right) \geq \mathcal{E}\left(\sum_{i \in V(G)} Z_{i}^{2}\right)=\sum_{i \in V(G)} \mathcal{E}\left(Z_{i}^{2}\right) \\
& =\sum_{i \in V(G)} \sum_{k=1}^{d_{i}} \frac{1}{1+k} P(i \in X \text { and }|N(i) \cap X|=k) \\
& =\sum_{i \in V(G)} \sum_{k=1}^{d_{i}} \frac{1}{1+k} P(i \in X) P(|N(i) \cap X|=k) \\
& =\sum_{i \in V(G)} x_{i} \sum_{k=1}^{d_{i}} \frac{1}{1+k} P(|N(i) \cap X|=k) . \\
& P(|N(i) \cap X|=0)+\sum_{k=1}^{d_{i}} P(|N(i) \cap X|=k)=1 \text { for } i \in V(G) \text { and with } \\
& \mu_{i}=P(|N(i) \cap X|=0)=\prod_{j \in N(i)}\left(1-x_{j}\right) \text { and } \sigma_{i k}=P(|N(i) \cap X|=k), \\
& \mathcal{E}\left(\alpha\left(H_{2}\right)\right) \geq \sum_{i \in V(G)} x_{i} \sum_{k=1}^{d_{i}} \frac{1}{1+k} \sigma_{i k}=\sum_{i \in V(G), \mu_{i}<1} x_{i} \sum_{k=1}^{d_{i}} \frac{1}{1+k} \sigma_{i k} \\
& =\sum_{i \in V^{\prime}} x_{i} \sum_{k=1}^{d_{i}} \frac{1}{1+k} \sigma_{i k}=\sum_{i \in V^{\prime}} x_{i}\left(1-\mu_{i}\right) \sum_{k=1}^{d_{i}} \frac{\sigma_{i k}}{(1+k)\left(1-\mu_{i}\right)} .
\end{aligned}
$$

For $\lambda_{i k}=\frac{\sigma_{i k}}{1-\mu_{i}}$ we have $\lambda_{i k} \geq 0, \sum_{k=1}^{d_{i}} \lambda_{i k}=1$ if $i \in V^{\prime}$, and again using Jensen's inequality,

$$
\begin{aligned}
& \mathcal{E}\left(\alpha\left(H_{2}\right)\right) \geq \sum_{i \in V^{\prime}} x_{i}\left(1-\mu_{i}\right) \frac{1}{1+\sum_{k=1}^{d_{i}} k \lambda_{i k}} \\
& =\sum_{i \in V^{\prime}} \frac{x_{i}\left(1-\prod_{j \in N(i)}\left(1-x_{j}\right)\right)^{2}}{1-\prod_{j \in N(i)}\left(1-x_{j}\right)+\sum_{k=1}^{d_{i}} k P(|N(i) \cap X|=k)}=\sum_{i \in V^{\prime}} \frac{x_{i}\left(1-\prod_{j \in N(i)}\left(1-x_{j}\right)\right)^{2}}{1-\prod_{j \in N(i)}\left(1-x_{j}\right)+\sum_{j \in N(i)} x_{j}} .
\end{aligned}
$$

Finally, let us consider the random variable $Z_{i}^{3}$ with $Z_{i}^{3}=\frac{1}{1+k}$ if $i \in V\left(H_{3}\right)$ and $\left|N(i) \cap V\left(H_{3}\right)\right|=k \geq 0$, and $Z_{i}^{3}=0$ if $i \notin V\left(H_{3}\right)$. Then

$$
\begin{aligned}
& \mathcal{E}\left(\alpha\left(H_{3}\right)\right) \geq \mathcal{E}\left(\sum_{i \in V(G)} Z_{i}^{3}\right)=\sum_{i \in V(G)} \mathcal{E}\left(Z_{i}^{3}\right) \\
& =\sum i \in V(G) \sum_{k=0}^{d_{i}} \frac{1}{1+k} P\left(i \in V\left(H_{3}\right) \text { and }\left|N(i) \cap V\left(H_{3}\right)\right|=k\right) \\
& =\sum_{i \in V(G)} \sum_{k=0}^{d_{i}} \frac{1}{1+k} P\left(i \in V\left(H_{3}\right)\right) P\left(\left|N(i) \cap V\left(H_{3}\right)\right|=k \mid i \in V\left(H_{3}\right)\right) \\
& =\sum_{i \in V(G)}\left(\left(1-x_{i}\right) \prod_{j \in N(i)}\left(1-x_{j}\right) \sum_{k=0}^{d_{i}} \frac{1}{1+k} P\left(\left|N(i) \cap V\left(H_{3}\right)\right|=k \mid i \in V\left(H_{3}\right)\right)\right)
\end{aligned}
$$




$$
\begin{aligned}
& \geq \sum_{i \in V(G)}\left(\left(1-x_{i}\right) \prod_{j \in N(i)}\left(1-x_{j}\right) \frac{1}{1+\sum_{k=0}^{d_{i}} k P\left(\left|N(i) \cap V\left(H_{3}\right)\right|=k \mid i \in V\left(H_{3}\right)\right)}\right) \\
& =\sum_{i \in V(G)}\left(\left(1-x_{i}\right) \prod_{j \in N(i)}\left(1-x_{j}\right) \frac{1}{1+\sum_{j \in N(i)} \prod_{l \in N(j) \backslash(N(i) \cup\{i\})}\left(1-x_{l}\right)}\right),
\end{aligned}
$$

and Lemma 3 is proved.

Theorem 4, 5, and 6 follow with $\mathcal{E}(|X|)=\sum_{i \in V(G)} x_{i}, \mathcal{E}\left(\left|E\left(H_{2}\right)\right|\right)$

$=\sum_{i j \in E(G)} x_{i} x_{j}, \mathcal{E}\left(\left|V\left(H_{2}\right)\right|\right)=\sum_{i \in V(G)} x_{i}\left(1-\prod_{j \in N(i)}\left(1-x_{j}\right)\right)$, Lemma 1, 2, and 3.

\section{Remarks}

For $\phi, \psi \in\{e, f, g, h\}$ define $\phi \leq \psi$ if $\phi_{G}\left(x_{1}, x_{2}, \ldots, x_{n}\right) \leq \psi_{G}\left(x_{1}, x_{2}, \ldots, x_{n}\right)$ for every graph $G$ on $n$ vertices and for every $\left(x_{1}, x_{2}, \ldots, x_{n}\right) \in C^{n}$. We write $\phi<>\psi$ if neither $\phi \leq \psi$ nor $\psi \leq \phi$.

Remark 1. $h \leq f \leq g, e \leq g$ and $e<>f$.

Proof. We will use the following Lemma 4, which can be seen easily by induction on $r$.

Lemma 4. For an integer $r \geq 1$ and $a_{1}, a_{2}, \ldots, a_{r} \in[0,1]$, $\sum_{q=1}^{r} a_{q}+\prod_{q=1}^{r}\left(1-a_{q}\right) \geq 1$.

The inequality $h \leq f$ is obvious. To see $f \leq g$, first notice that $\sum_{i \in V(G)} x_{i}-\sum_{i j \in E(G)} x_{i} x_{j}=\sum_{i \in V(G)} x_{i}\left(1-\frac{1}{2} \sum_{j \in N(i)} x_{j}\right)$. If $\sum_{j \in N(i)} x_{j}=0$ for an $i \in V(G)$ then $x_{i}=x_{i}\left(\prod_{j \in N(i)}\left(1-x_{j}\right)\right)=x_{i}\left(1-\frac{1}{2} \sum_{j \in N(i)} x_{j}\right)$. Hence, with the abbreviation $\mu_{i}=\prod_{j \in N(i)}\left(1-x_{j}\right)$ and $\rho_{i}=\sum_{j \in N(i)} x_{j}$ for $i \in V(G)$ we have to show $\sum_{i \in V^{\prime}}\left(x_{i}\left(\mu_{i}+\frac{\left(1-\mu_{i}\right)^{2}}{1-\mu_{i}+\rho_{i}}\right)\right) \geq \sum_{i \in V^{\prime}}\left(x_{i}\left(1-\frac{1}{2} \rho_{i}\right)\right)$, where again $V^{\prime}=\left\{i \in V(G) \mid \sum_{j \in N(i)} x_{j}>0\right\}$.

Using Lemma 4 , even $\mu_{i}+\frac{\left(1-\mu_{i}\right)^{2}}{1-\mu_{i}+\rho_{i}} \geq 1-\frac{1}{2} \rho_{i}$ for all $i \in V^{\prime}$.

To prove $e \leq g$, we have to show 
$\sum_{i \in V(G)} \frac{x_{i}}{1+\sum_{j \in N(i)} x_{j}} \leq \sum_{i \in V(G)} x_{i} \prod_{j \in N(i)}\left(1-x_{j}\right)+\sum_{i \in V^{\prime}} \frac{x_{i}\left(1-\prod_{j \in N(i)}\left(1-x_{j}\right)\right)^{2}}{1-\prod_{j \in N(i)}\left(1-x_{j}\right)+\sum_{j \in N(i)} x_{j}}$.

Since $\frac{x_{i}}{1+\sum_{j \in N(i)} x_{j}}=x_{i} \prod_{j \in N(i)}\left(1-x_{j}\right)$ if $\sum_{j \in N(i)} x_{j}=0$, it is sufficient to establish $\frac{1}{1+\rho_{i}} \leq \mu_{i}+\frac{\left(1-\mu_{i}\right)^{2}}{1-\mu_{i}+\rho_{i}}$ if $\sum_{j \in N(i)} x_{j}>0$, what is verified easily.

For a cycle $C_{n}$ on $n$ vertices $e_{C_{n}}\left(\frac{1}{3}, \frac{1}{3}, \ldots, \frac{1}{3}\right)<f_{C_{n}}\left(\frac{1}{3}, \frac{1}{3}, \ldots, \frac{1}{3}\right), e_{C_{n}}\left(\frac{2}{3}, \frac{2}{3}, \ldots, \frac{2}{3}\right)$ $>f_{C_{n}}\left(\frac{2}{3}, \frac{2}{3}, \ldots, \frac{2}{3}\right)$ and Remark 1 is proved.

With $h \leq f$ and $e<>f$ it is clear that $e \leq h$ does not hold. It remains open, whether $h<>e$ or $h \leq e$.

Theorems 1, 2, 4, 5, 6, and 7 are of that type that the independence number $\alpha(G)$ of a graph $G$ on $n$ vertices equals the optimum value of a continuous optimization problem $O(G)$ to maximize a certain function $\phi_{G}$ over $C^{n}$. Hence, $\phi_{G}\left(x_{1}, x_{2}, \ldots, x_{n}\right)$ is a lower bound on $\alpha(G)$ for every $\left(x_{1}, x_{2}, \ldots, x_{n}\right) \in$ $C^{n}$. Let $\left(x_{1}^{\prime}, x_{2}^{\prime}, \ldots, x_{n}^{\prime}\right) \in C^{n}$ be the solution of an arbitrary approximation algorithm for $O(G)$. How to find an independent set $I$ of $G$ in polynomial time such that $|I| \geq \phi_{G}\left(x_{1}^{\prime}, x_{2}^{\prime}, \ldots, x_{n}^{\prime}\right)$ ? In [8] and [9] efficient algorithms forming $I$ with $|I| \geq \phi_{G}\left(x_{1}^{\prime}, x_{2}^{\prime}, \ldots, x_{n}^{\prime}\right)$ are given if $O(G)$ is the optimization problem of Theorem 1 or of Theorem 2. Remark 2 shows that this is also possible if we consider the case $\phi_{G}=h_{G}$. In case $\phi_{G}=e_{G}, \phi_{G}=f_{G}$ or $\phi_{G}=g_{G}$ the problem remains open, whether such an algorithm exists.

Remark 2. There is an $\mathcal{O}(\Delta(G) n)$-algorithm with

INPUT: $\left(x_{1}, x_{2}, \ldots, x_{n}\right) \in C^{n}$,

OUTPUT: an independent set $I \subseteq V(G)$ with $|I| \geq \sum_{i \in V(G)} x_{i}-\sum_{i j \in E(G)} x_{i} x_{j}$.

Proof. First we give the Algorithm:

1. For $i=1$ to $n$ do if $\sum_{j \in N(i)} x_{j}<1$ then $x_{i}:=1$ else $x_{i}:=0$.

2. For $i=1$ to $n$ do if $\left(x_{i}=1\right.$ and $\left.\prod_{j \in N(i)}\left(1-x_{j}\right)=0\right)$ then $x_{i}:=0$.

3. $I:=\left\{i \in V(G) \mid x_{i}=1\right\}$.

\section{STOP}

It is obvious that the algorithm in an $\mathcal{O}(\Delta(G) n)$-algorithm. For the input vector $\left(x_{1}, x_{2}, \ldots, x_{n}\right) \in C^{n}$ set

$$
\sum_{k \in V(G)} x_{k}-\sum_{k j \in E(G)} x_{k} x_{j}=a .
$$


After step 1 , the current $\left(x_{1}, x_{2}, \ldots, x_{n}\right)$ is a 0 -1-vector and

$$
\sum_{k \in V(G)} x_{k}-\sum_{k j \in E(G)} x_{k} x_{j} \geq a
$$

because

$\frac{\partial}{\partial x_{i}}\left(\sum_{k \in V(G)} x_{k}-\sum_{k j \in E(G)} x_{k} x_{j}\right)=1-\sum_{j \in N(i)} x_{j}$, i.e., $\sum_{k \in V(G)} x_{k}-\sum_{k j \in E(G)} x_{k} x_{j}$

is multilinear.

In step 2, $\prod_{j \in N(i)}\left(1-x_{j}\right)=0$ if and only if there is at least one $j \in$ $N(i)$ such that $x_{j}=1$. With $x_{i}=0$ instead of $x_{i}=1$ the sum $\sum_{k \in V(G)} x_{k}$ decreases by 1 and the sum $\sum_{k j \in E(G)} x_{k} x_{j}$ decreases by at least 1 , hence $\sum_{k \in V(G)} x_{k}-\sum_{k j \in E(G)} x_{k} x_{j}$ does not decrease.

After step 2,

$x_{k} x_{j}=0$ for all $k j \in E(G),|I|=\sum_{k \in V(G)} x_{k}=\sum_{k \in V(G)} x_{k}-\sum_{k j \in E(G)} x_{k} x_{j} \geq a$ and Remark 2 is proved.

\section{References}

[1] E. Bertram, P. Horak, Lower bounds on the independence number, Geombinatorics, (V) 3 (1996) 93-98.

[2] R. Boppana, M.M. Halldorsson, Approximating maximum independent sets by excluding subgraphs, BIT 32 (1992) 180-196.

[3] Y. Caro, New results on the independence number (Technical Report, Tel-Aviv University, 1979).

[4] S. Fajtlowicz, On the size of independent sets in graphs, in: Proc. 9th S-E Conf. on Combinatorics, Graph Theory and Computing (Boca Raton 1978) 269-274.

[5] S. Fajtlowicz, Independence, clique size and maximum degree, Combinatorica 4 (1984) 35-38.

[6] M.R. Garey, D.S. Johnson, Computers and Intractability, A Guide to the Theory of NP-Completeness (W.H. Freeman and Company, San Francisco, 1979). 
[7] M.M. Halldorsson, J. Radhakrishnan, Greed is good: Approximating independent sets in sparse and bounded-degree graphs, Algorithmica 18 (1997) $145-163$.

[8] J. Harant, A lower bound on the independence number of a graph, Discrete Math. 188 (1998) 239-243.

[9] J. Harant, A. Pruchnewski, M. Voigt, On dominating sets and independent sets of graphs, Combinatorics, Probability and Computing 8 (1999) 547-553.

[10] J. Harant, I. Schiermeyer, On the independence number of a graph in terms of order and size, submitted.

[11] T.S. Motzkin, E.G. Straus, Maxima for graphs and a new proof of a theorem of Turán, Canad. J. Math. 17 (1965) 533-540.

[12] O. Murphy, Lower bounds on the stability number of graphs computed in terms of degrees, Discrete Math. 90 (1991) 207-211.

[13] S.M. Selkow, The independence number of graphs in terms of degrees, Discrete Math. 122 (1993) 343-348.

[14] S.M. Selkow, A probabilistic lower bound on the independence number of graphs, Discrete Math. 132 (1994) 363-365.

[15] J.B. Shearer, A note on the independence number of triangle-free graphs, Discrete Math. 46 (1983) 83-87.

[16] J.B. Shearer, A note on the independence number of triangle-free graphs, II, J. Combin. Theory (B) 53 (1991) 300-307.

[17] V.K. Wei, A lower bound on the stability number of a simple graph (Bell Laboratories Technical Memorandum 81-11217-9, Murray Hill, NJ, 1981).

Received 8 February 1999 